\title{
The Last Mile: Using Fax Machines to Exchange Data between Clinicians and Public Health
}

\author{
Stephen M. Downs, MD, MS, ${ }^{1,2}$ Vibha Anand, PhD, ${ }^{1.2}$, Meena Sheley, ${ }^{1} \mathrm{BS}$, \\ Shaun J. Grannis, MD, MS \\ ${ }^{1}$ Children's Health Services Research, Indiana University \\ ${ }^{2}$ Regenstrief Institute, Inc. Indianapolis, IN
}

Abstract

There is overlap in a wide range of activities to support both public health and clinical care. Examples include immunization registries (IR), newborn screening (NBS), disease reporting, lead screening programs, and more. Health information exchanges create an opportunity to share data between the clinical and public health environments, providing decision support to clinicians and surveillance and tracking information to public health. We developed mechanisms to support two-way communication between clinicians in the Indiana Health information Exchange (IHIE) and the Indiana State Department of Health (ISDH). This paper describes challenges we faced and design decisions made to overcome them.

We developed systems to help clinicians communicate with the ISDH IR and with the NBS program. Challenges included (1) a minority of clinicians who use electronic health records (EHR), (2) lack of universal patient identifiers, (3) identifying physicians responsible for newborns, and (4) designing around complex security policies and firewalls.

To communicate electronically with clinicians without EHRs, we utilize their fax machines. Our rule-based decision support system generates tailored forms that are automatically faxed to clinicians. The forms include coded input fields that capture data for automatic transfer into the IHIE when they are faxed back. Because the same individuals have different identifiers, and newborns' names change, it is challenging to match patients across systems. We use a stochastic matching algorithm to link records. We scan electronic clinical messages (HL7 format) coming into IHIE to find clinicians responsible for newborns. We have designed an architecture to link IHIE, ISDH, and our systems.

Key Words: newborn, screening, informatics, public health, immunization, registry health information exchange

\section{Introduction}

A major challenge for public health informatics is facilitating the exchange of information between public health and clinical care. Data in public health information systems often come 
from forms filled out by hand, which are later computer-coded. Even when reporting is electronic, initial data entry is typically still manual. As a result reportable diseases and conditions may be underreported. ${ }^{1}$ Data need to flow automatically to public health from clinical environments. When these data are appropriately compiled by public health information systems, they can allow more rapid and accurate assessments and disease control responses, as well as the formulation of improved clinical guidelines and interventions. Conversely, automated presentation to clinicians of prevention guidelines has been shown to improve clinical care. ${ }^{2}$ There are numerous ways in which the skills and activities of the public health community could benefit clinical care. Electronic information sharing is a means by which we believe public health and clinical care activities can be integrated. ${ }^{3}$

This is especially important in children, who undergo a series of preventive and therapeutic health interventions and activities that are relevant to public health, including newborn screening, immunizations, and lead screening. Typically, each activity includes collection and submission of data to a dedicated public health system. ${ }^{4}$ Programs that can integrate decision support and data capture into the clinical workflow are likely to improve these shared functions.

For example, newborn screening programs improve outcomes and are among the most costeffective (even cost saving) strategies in the healthcare system. ${ }^{5}$ Effective newborn screening programs consist of not only the screening tests, but also confirmation and assurance of appropriate treatment and follow-up of identified conditions. Primary care physicians play an important role in the success of the newborn screening system and should have appropriate ongoing involvement in follow-up and tracking efforts. Most pediatricians believe that primary care physicians should be responsible for informing families about a positive newborn screen, arranging confirmatory testing, and coordinating subspecialty referral. However, many do not feel competent to discuss conditions included in newborn screening panels, ${ }^{6}$ which emphasize the need for decision support.

Likewise, immunizations are one of the most successful and effective public health tools for preventing disease, disability, and death from preventable disease. ${ }^{7}$ The American Academy of Pediatrics (AAP) and the Advisory Committee on Immunization Practices (ACIP) of the Centers for Disease Control and Prevention (CDC) have provided national standard guidelines for immunization and have constantly updated them since $1997 .{ }^{8}$ To maximize vaccination rates, immunization registries have been developed in most states to track the complex series of vaccines that may be delivered by a variety of providers. However, registries never really reach their potential unless they are utilized by the vast majority of clinicians who provide immunizations. While $75 \%$ of children have vaccine data in registries, and registries are widespread and capable of sharing data, only a minority of providers routinely access them, ${ }^{9}$ largely because only $3 \%$ of pediatricians use electronic medical records (EMR) that are both fully functional and pediatric-appropriate. ${ }^{10}$ This may be because so few systems are specifically designed with pediatric needs in mind.

Even among those who have an EMR, most of their systems do not provide clinical decision support. One survey of 1000 primary care pediatricians ${ }^{11}$ found only $21 \%$ used electronic health records. Among those that had EMR, a paltry $49 \%$ have preventive services prompts, and only $33 \%$ provided alerts for abnormal results. Pediatricians who did not use EMRs cited two 
significant barriers to EMR implementation. The first, cost, was identified by $94 \%$ and was especially problematic for small practices. Interestingly, $58 \%$ perceived no potential improvement in care from an EMR.

Clearly there is a need for electronic communications between public health systems and clinicians. Moreover, clinicians would benefit from decision support in the areas of immunization forecasting and management of conditions detected by newborn screening. These needs are reflected in the "meaningful use" rules set forth by the Office of the National Coordinator for Health Information Technology. ${ }^{12}$ However, until there is more widespread adoption of fully functional EMR technology in pediatrics practices, other strategies of connecting public health to pediatric practitioners will be needed. In Indiana, we are leveraging a statewide health information exchange ${ }^{13}$ to explore methods for connecting public health systems and decision support to clinicians who may have no technology more elaborate that a fax machine.

\section{Methods}

Our approach builds on two initiatives - the Indiana Health Information Exchange (IHIE) and the Child Health Improvement through Computer Automation system (CHICA). By monitoring messages entering IHIE from the state health department and from the health systems in the exchange, we are able to link clinicians with public health. Using rule based logic, our system can deliver decision support to both clinicians and the health department. Finally, using a tailored scannable paper as an interface with optical character recognition (OCR) and optical mark recognition (OMR), we can capture coded data from clinicians with nothing more than a fax machine.

\section{IHIE and the Indiana Network for Patient Care}

The Indiana Health Information Exchange operates the nation's largest health information exchange, partnering with communities throughout Indiana. ${ }^{14}$ IHIE connects hospitals, rehabilitation centers, long term care facilities, laboratories, imaging centers, clinics, community health centers and other healthcare organizations. Data are transmitted from these sources by HL7 messaging and stored in separate files within one data repository known as the Indiana Network for Patient Care (INPC). ${ }^{3}$

The INPC receives data from 70 hospitals and 18,000 physicians. It contains data from over 11 million patients with almost 24 million patient registrations and 3.8 billion clinical results. ${ }^{14}$ IHIE operates a results delivery system, DOCS4DOCS, ${ }^{15}$ which has delivered over 77 million clinical results to participating clinicians. These results can be delivered to an EMR, to a secure electronic mailbox or to a fax machine.

\section{CHICA and Adaptive Turnaround Documents}

To leverage the health information exchange and the results delivery system, we needed a decision support engine that could interpret inbound HL7 messages from different sources and generate appropriate information to deliver to the health department and the appropriate clinician. We started with the framework of the Child Health Improvement through Computer Automation (CHICA) system. ${ }^{16}$ 
CHICA is a clinical decision support system for pediatric primary care that has been running in pediatric clinics in Indiana for seven years. The system has an HL7 message processor that allows it to communicate with its underlying EMR, the Regenstrief Medical Record System (RMRS). It is built on an open source medical record framework known as OpenMRS. ${ }^{17,18}$ CHICA has a logic module that interprets rules encoded in Arden Syntax. ${ }^{19}$ These rules (or medical logic modules, MLMs) can extract and interpret data in the underlying medical record and write new data into the record.

One of CHICA's most unusual features is its user interface, which consist of tailored, scannable forms printed on paper. We refer to these forms as Adaptive Turn Around Documents (ATAD). ATAD forms are printed and scanned using the Teleform desktop suite version 10.x (www.verity.com) that includes an AutoMerge Publisher, an OCR/OMR Reader and a Verifier. The AutoMerge Publisher populates a form template (to be completed by the patient or physician) with patient specific questions or prompts that have been generated by the system with Arden MLMs. The forms are then printed by a standard laser printer. Data entry is achieved by scanning a patient questionnaire form (completed by the family in the waiting room) or the physician worksheet using a standard document scanner. The system also stores a TIFF image of the scanned forms. The Teleform Reader interprets the handwritten numbers and checkbox responses.

\section{Enhancing the State Newborn Screening Program}

The ATAD model allows data to be captured from facsimile (fax) documents as well as scanned documents. With this capability, we wanted to test the feasibility of using ATADs to link newborn screening (NBS) programs, subspecialists and the medical home. The system is intended to enhance the NBS process in three ways. First, it provides just-in-time information to the medical home (physicians and families). Second, it can prevent missed opportunities to screen by identifying children with medical encounters anywhere in the state for whom no NBS result is known. Finally, it would facilitate long term tracking of children with identified conditions. The approach utilizes IHIE, DOCS4DOCS and CHICA in the following steps.

First, we expanded the basic CHICA architecture with a fax server (FaxPress 2500, OpenText, Bellevue, WA, faxsolutions.opentext.com), a device that enables us to send and receive faxes from any device on the network. Thus, the fax server can receive a completed ATAD from a fax machine anywhere and render it as a TIFF image that the TeleForm software can interpret just as it does the scanned images in the basic CHICA implementation.

The newborn screening laboratory, under contract with the ISDH, conducts metabolic screening on dried blood spots, compiles the results, combines them with newborn hearing screening results, and sends them to ISDH. We have worked with ISDH to establish an HL7 version 2.x standard for packaging the results in messages that can be sent to the INPC. These HL7 messages are captured by our New Born Screening System (NBSS), a software instantiation similar to CHICA.

The HL7 message is parsed, and a stochastic matching algorithm (see below) matches the message against records in the OpenMRS database. If the result matches an existing patient, the 
record is appended. If it is a new patient, a new record is created. Early manual testing of this stochastic algorithm show it to be highly reliable, and the production system does not require manual checking. Often, duplicate HL7 messages are sent. Our system recognizes and eliminates these in two steps. First, a numeric hash function is applied to each HL7 message. Then the resulting hash value is compared among messages, and exact duplicates are eliminated.

When an HL7 message arrives, the system invokes the Arden Syntax rules to interpret the NBS results contained in the message. If the results are abnormal and require further diagnosis and treatment or if the specimen is inadequate and the screen needs to be repeated, CHICA generates an ATAD. (Figure 1) These ATADs incorporate the American College of Medical Genetics ACTion Sheets. ${ }^{20}$ These sheets provide information about the condition, confirmatory diagnosis, and emergency management.

Among the biggest challenges NBS programs face is the identification of the physician who has responsibility for an identified child. Another challenge is finding children in the community who have not been screened. To meet these challenges, the system parses all incoming HL7 messages related to any child under the age of one month anywhere in the INPC. These may include admission or discharge messages, laboratory result messages, etc. Each of these messages is parsed and matched against our database of NBS results. If the child is identified in the system, the Arden rules determine if there was a normal newborn screen result in the database. If so, no further action is needed. If there is an abnormal or missing result, an appropriate ATAD is generated.

Matching records presented a challenge because data identifying newborns is highly variable among systems and across time. For example, at birth a child's name may be recorded as "Baby Boy Smith" because his mother's name is Smith. However, in a week's time, the name may be "Matthew Jones, Jr.," after his father. To match such variable records across systems requires a matching algorithm that is more sophisticated than most. We utilize a probabilistic matching algorithm developed in our group. ${ }^{21}$ The matching algorithm is based on the information theory concept of entropy and utilizes patient identifying data in the patient identification (PID) and next of kin (NK1) segments of the HL7 messages. The PID segment includes information about the patient such as name, date of birth, sex, race, address and phone number. The NK1 segment contains similar information about the baby's mother. Our work shows that comparing these using a statistical algorithm produces highly accurate matches even when some data are missing or changed. ${ }^{21}$ 


\section{Jennifer D. Patient}

DOB: 14 May 2007

Newborn Screening Alert: Elevated C8 with Lesser Elevations of C6 and C10 Acylcarnitine Suggestive of Medium-chain Acyl-CoA Dehydrogenase (MCAD) Deficiency

Condition Description: MCAD deficiency is a fatty acid oxidation (FAO) disorder. FAO occurs during prolonged fasting and/or periods of increased energy demands (fever, stress) when energy production relies increasingly on fat metabolism. In an FAO disorder, fatty acids and potentially toxic derivatives accumulate because of a deficiency in one of the mitochondrial FAO enzymes.

\section{MEDICAL EMERGENCY - TAKE THE FOLLOWING IMMEDIATE ACTIONS:}

- Contact family to inform them of the newborn screening result and ascertain clinical status (poor feeding, vomiting, lethargy).

- Consult with pediatric metabolic specialist.

- Evaluate the newborn (poor feeding, lethargy, hypotonia, hepatomegaly). If signs are present or infant is ill, initiate emergency treatment with IV glucose. Transport to hospital for further treatment in consultation with metabolic specialist. If infant is normal initiate timely confirmatory/diagnostic testing, as recommended by specialist.

- Educate family about need for infant to avoid fasting. Even if mildly ill, immediate treatment with IV glucose is needed.

- Report findings to newborn screening program.

Diagnostic Evaluation: Plasma acylcarnitine analysis will show elevated octanoylcarnitine (C8). Urine acylglycine will show elevated hexanoylglycine. Diagnosis is confirmed by mutation analysis of the MCAD gene.

\section{Please check ALL of the following that apply:}

\section{[ ] Family contacted}

[ ] Newborn clinical status assessed

[ ] Problems (poor feeding, vomiting, lethargy, hypotonia, hepatomegaly)

$\rightarrow$ [ ] Treated with IV glucose

[ ] Infant stable

\section{[ ] Family provided attached educational materials}

\section{Diagnostic Evaluation}

[ ] Plasma acylcarnitine sent

[ ] Referral made to metabolic center

\section{[ ] Family could not be contacted}

[ ] This is not my patient

Figure 1: An example of an adaptive turnaround document about a child with an abnormal newborn screen delivered to a primary care clinician using the DOCS4DOCS system 


\section{Linking Practices to the Immunization Registry}

A second application of the CHICA model to link clinicians to public health systems is the CHICA Immunization Assistant (CHIA). CHIA helps facilitate participation in an immunization information system (IIS or registry) by clinicians who lack EMR systems or access to the Internet (because of technical or time constraints). As with the NBS module, the concept is to provide an interface that requires minimal technology - a fax machine - and will fit into the clinic workflow.

The ISDH has a well developed statewide IIS known as the Children and Hoosier Immunization Registry Project (CHIRP). CHIRP offers a web application that allows enrolled users to search for patients, view vaccination records, and add or edit patient records and vaccination records. (https://chirp.in.gov) CHIRP captures immunization data from all the Indiana Health Departments and from Medicaid claims. Unfortunately, use of CHIRP by pediatric practitioners has not been high. CHIRP provides an HL7 interface for EHR systems. ${ }^{22}$ However, because of the low penetration of comprehensive EHRs among pediatricians, ${ }^{10}$ a new solution was needed.

CHIA serves as a "low tech" front end to CHIRP. Equipment required by the practice to use CHIA will be only a fax machine and scannable forms. In order for a physician to enroll a patient through CHIA, a form is completed and faxed. This form has spaces for the patient's name, date of birth, mother's name, address, phone number, social security number, and membership number in the US Department of Agriculture Women, Infants and Children (WIC) program. The clinic completes whatever fields on the form are available and faxes it to the CHIA toll-free number.

The fax is received by the CHIA fax server, and the coded fields from the faxed form are extracted and read using OCR by Teleform into the system's data tables. OCR accuracy is heavily dependent on handwriting, fax quality, and whether the field is numeric or alpha numeric. The system is in an early pilot phase, and several prototypes and revisions will be required to achieve appropriate settings for the software which has sensitivity and specificity settings that can be changed.

Once the data are extracted from the form, CHIA generates an HL7 vaccination record query (VXQ) message that is sent to CHIRP. In our implementation, HL7 messages are passed through the Indiana Network for Patient Care (INPC), which maintains a Virtual Private Network (VPN) connection to CHIRP. The VXQ message contains patient identifying information. CHIRP matches this information against records in the registry and returns the vaccination record via an HL7 vaccine query response (VXR) or returns an HL7 response to vaccination query with multiple patient matches (VXX) message containing possible patient matches. In the case that no match is found, CHIA will create a record in CHIRP, using an unsolicited vaccination record (VXU) message. When more than one match is found, the clinician will be required to disambiguate.

When a patient match is achieved, CHIRP responds by faxing a form back to the clinician (Figure 2). This form contains a unique identifier, generated by CHIA, which is encoded in a bar code. This allows CHIA to recognize the form and the patient to whom it refers when it is faxed back. The form provides the vaccine data available in CHIRP and a recommendation for what 
vaccines should be given on that date. In addition, the form allows the clinician to record past vaccination dates that are missing from CHIRP and to indicate, by checking boxes, which vaccines were given. Once these forms are completed, they are faxed to the CHIA toll-free number. The data are transferred to the CHIRP database. The form remains for the clinician to use as part of the paper medical record. This obviates the need for duplicate data entry.

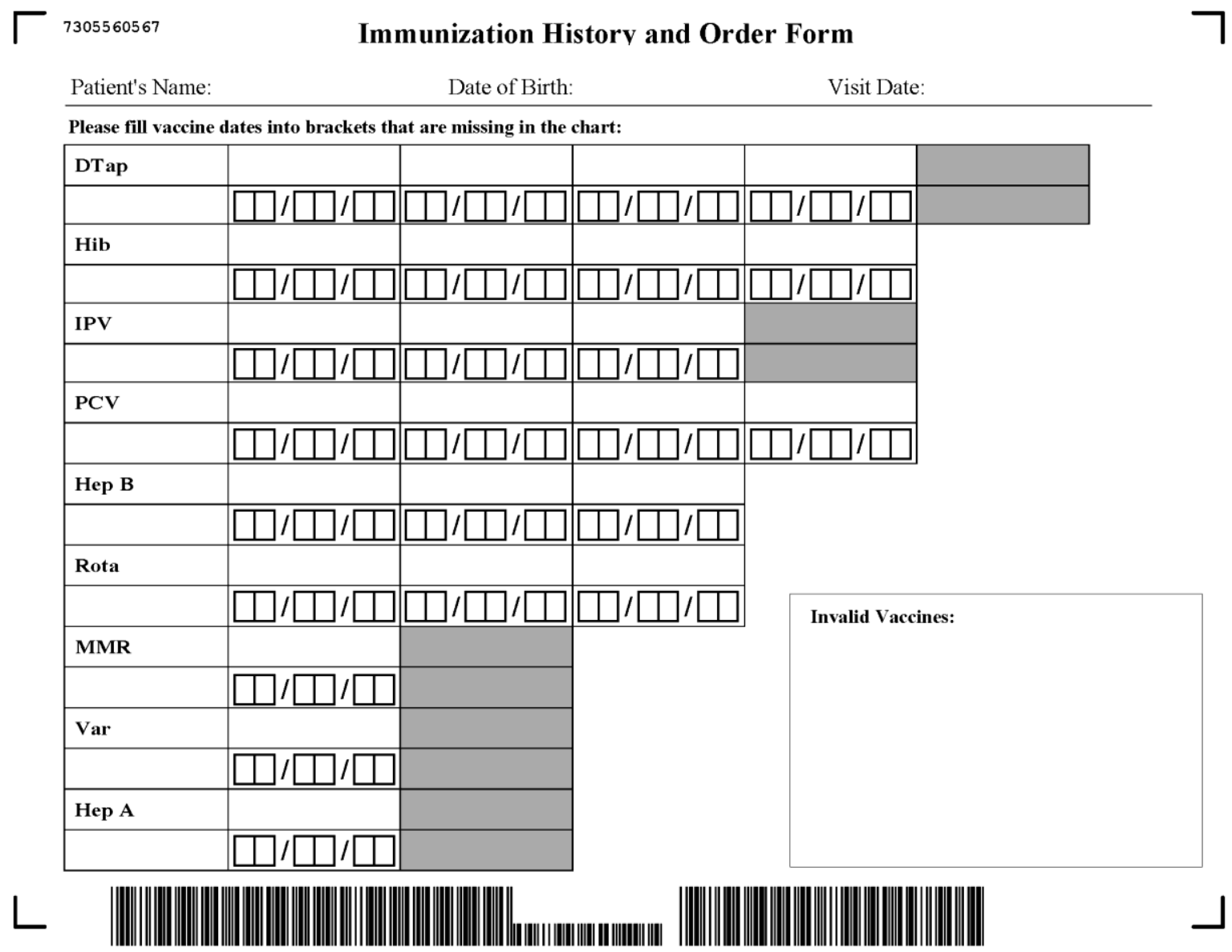

Page 1 of Fax 


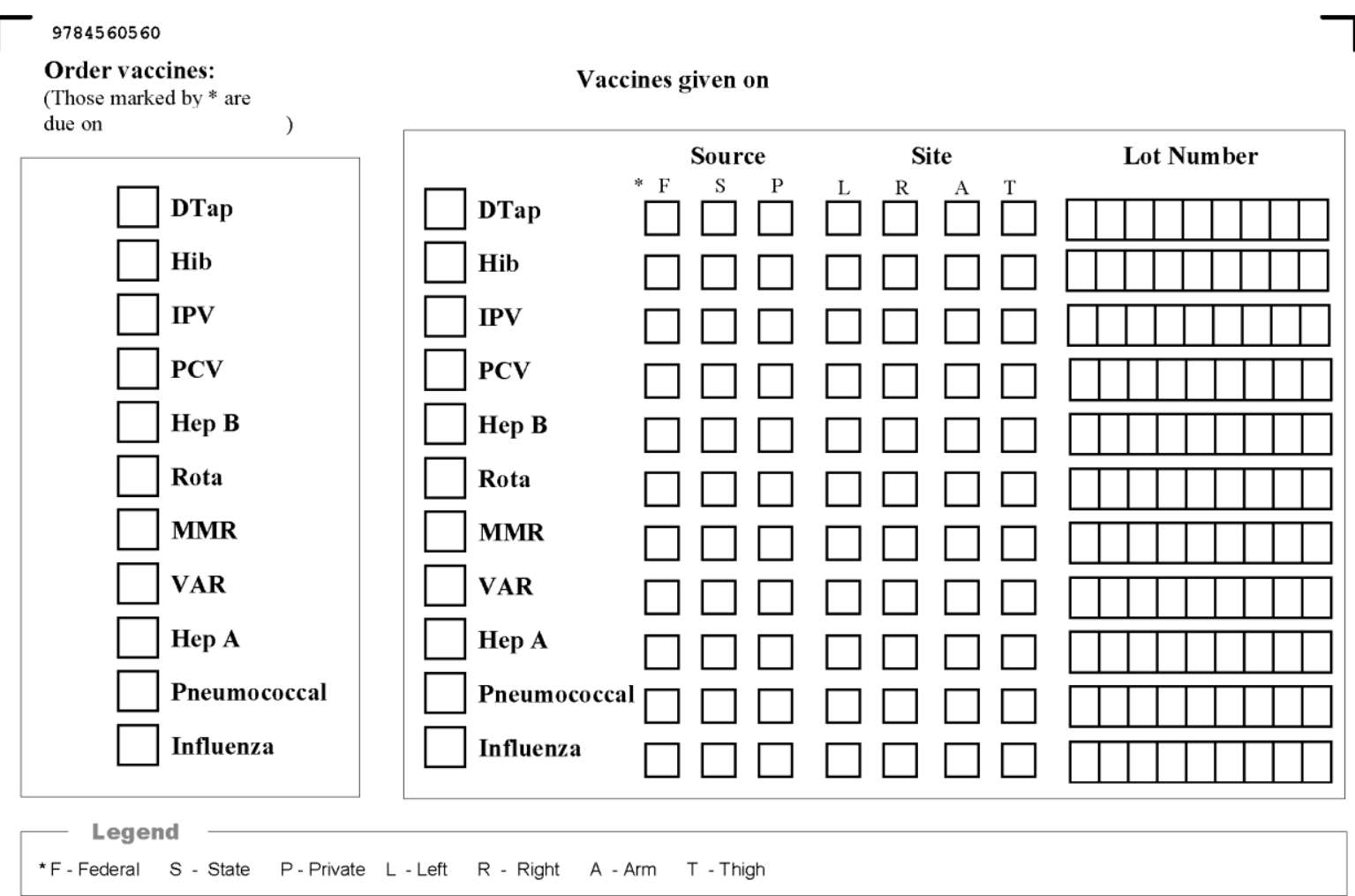

Parent's name (Print): Signature and Date:

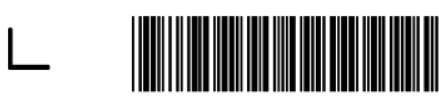

Figure 2: The CHIA immunization form used to transfer immunization data to and from the immunization registry by machine readable fax. Pages 1 and 2 shown

At subsequent encounters, the clinician can fax any of the existing paper forms completed for this patient because they all have the bar code that identifies the patient. Faxing a form will trigger the CHIA system to query CHIRP with another VXQ and capture the data from the registry. CHIA will fax back a new immunization form with any new vaccines and new recommendations.

\section{Results}

The project is in its preliminary, technical development stage. Described below are important milestones achieved to date as well as specific technical challenges and their solutions. 


\section{HL7 Transmission}

An early achievement was the establishment of a standard HL7 stream carrying newborn screening lab results. The output of the newborn screening laboratory consists of a set of proprietary results codes in a "pipe-delimited" file format. These are converted at the ISDH into standard HL7 version 2.x messages that are captured by our system. The results are still in a proprietary coding system but will, in time, be converted to a LOINC standard. ${ }^{23,24}$

An additional step was creating a filter that captures all HL7 messages entering the INPC for those whose patient identification (PID) segments show an age of 1 month or younger. These messages are packaged along with provider information (PV1 segments) and next of kin (NK1 segments) into an HL7 message that is passed on to the newborn screening system. We have demonstrated the process of parsing newborn screening lab messages, matching them against messages from the INPC, generating ATADs.

In an initial pilot test of the matching algorithm, we parsed 100,785 messages from the newborn screening laboratory. In the same timeframe, there were 2,561 HL7 messages entering the INPC for children of aged 1 month or younger. Thus, about $2.5 \%$ of newborns in Indiana will have a subsequent healthcare visit that appears in the INPC. Of the 2,561 INPC messages, 2,243 (88\%) could be linked to the messages from the newborn screening lab. This left 318 (12\%) of messages from the INPC that could not be tied to a newborn screening result. On manual review of these, most were "junk" messages that did not contain real patient data. Many were test messages or other information from external sources that have nothing to do with patients. However a small number (99) were patients who had not been screened. All but one of these were in neonatal intensive care units, a setting in which newborn screening can be missed. ${ }^{25}$

\section{Crossing Domains and Security Policies}

In order to deliver the messages to clinician participating in IHIE, we had to produce the ATADs and send them through the results delivery system, DOCS4DOCS. To do this the ATADs had to be converted to Portable Document Format (PDF), the only image format the system handles. This required additional software to make the translation. The forms were then placed in the OBX segment of an HL7 message and sent back to the INPC to be picked up by the DOCS4DOCS server and delivered.

Establishing servers in secure environment and establishing the interfaces with external systems (HL7 input and output feeds, fax server, Teleform) represented a significant technical hurdle. Figure 3 shows a schematic of the processes involved in the bringing the Teleform functionality, the CHICA rules engine, IHIE and the DOCS4DOCS services into one working system. Although the technical steps were facilitated by using health IT industry standards whenever feasible, negotiating each entities security policies required substantial effort.

\section{Handling Paper in an Electronic Environment}

We also found that the DOCS4DOCS results delivery system adds a header and footer to the images it delivers. The result is shrinkage of the image. Because the Teleforms software has to recognize bar codes and other markers on the forms, this posed a challenge. With experimentation and adoption of specific barcode formats, we found that the software could tolerate an image reduced by $15 \%$ or less and still scan reliably. 
When ATADs are completed and faxed back, the quality of the fax machine comes into play. Low quality machines can skew and distort the imagines, making machine recognition of the form and interpretation of the coded fields impossible. Knowing quality of forms we get back from the clinicians in the INPC will have to wait until full deployment of the system. However, manual review of critical forms may still be necessary. In our pilot program, data managers in the Children's Health Services Research section at Indiana University manually check faxes that are not interpretable by the OCR software.

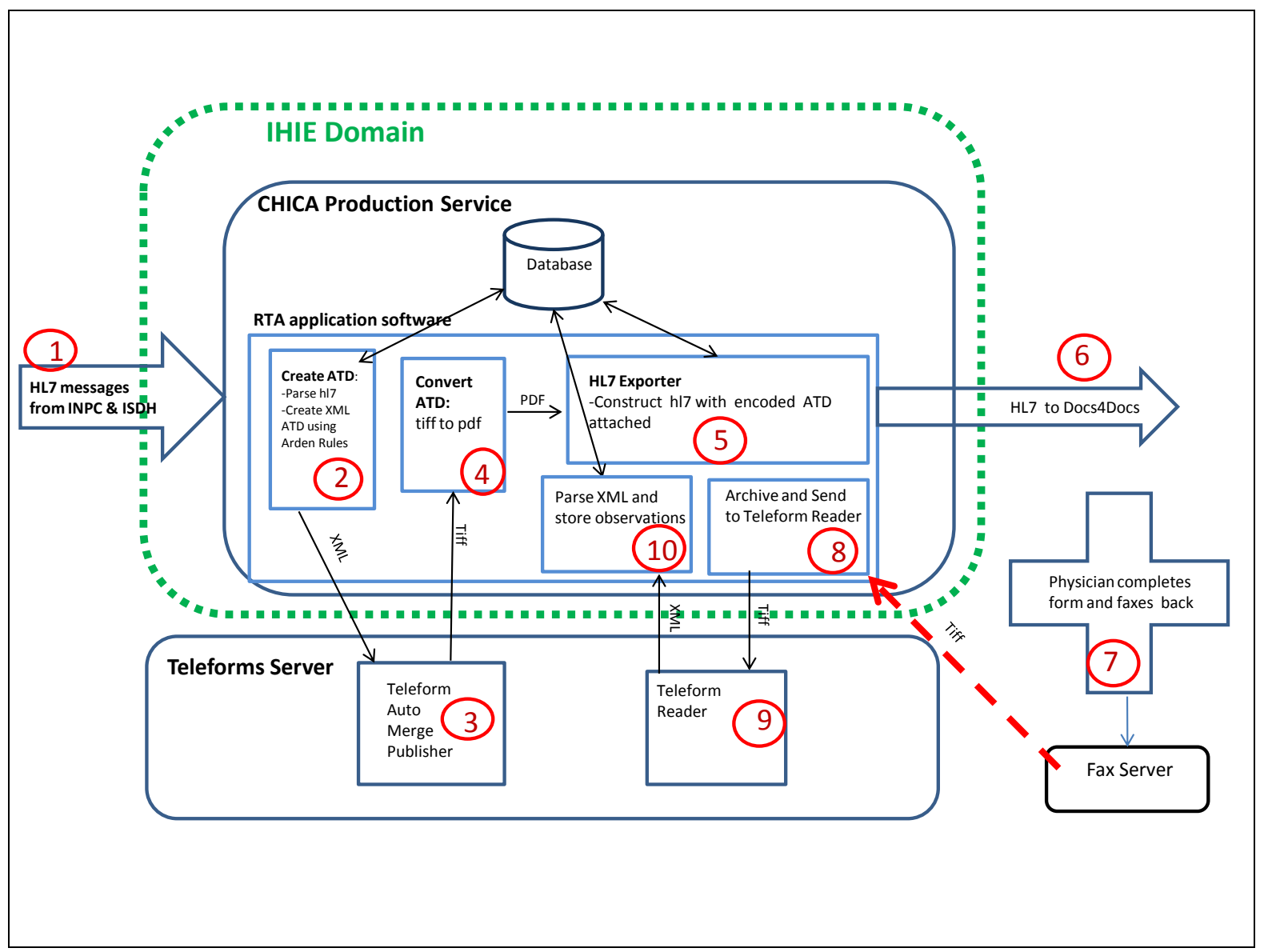

Figure 3: Complexities of connecting multiple systems across domains, firewalls and security policies 


\section{Discussion}

The potential benefits of linking public health functions to clinical practice seem obvious. The link between newborn screening programs and clinical follow-up is tenuous and highly variable from state to state,${ }^{26}$ and information systems offer a way to improve communication. Likewise, immunization rates are well below those needed to establish herd immunity, ${ }^{27}$ and immunization registries can have a significant effect, but only if there is adequate participation by clinicians in the community.

The Health Information Technology for Economic and Clinical Health (HITECH) Act includes "meaningful use" criteria and incentives for the meaningful adoption of health information technology, including linking EHRs to immunization registries. ${ }^{12}$ However, these incentives will have relatively less impact on pediatric care because they only apply to clinicians whose patient panels include minimal levels of Medicare or Medicaid enrollees. Because many pediatricians won't meet these levels, and the majority don't have comprehensive EMRs, the ATAD approach we are developing may be important for some time.

The advantage of our approach is that the clinician can share data with the public health community with only a fax machine and a phone line. However, this approach pushes the technical complexity upstream to entities running the health information exchange and the health department. The complexity of supporting a paper interface in an otherwise electronic health information exchange is substantial. For example, although most faxes can be interpreted by the software without human intervention, personnel with the skills to review faxes and manually enter data are needed if all of the data are to be captured.

Software systems for generating tailored paper forms and recognizing and interpreting those forms when returned by fax are complex, expensive and prone to errors. Moreover, our approach depends on the existence of the health information exchange and the results delivery system that can handle images such as our ATADs. Even when this infrastructure is in place, our programs had to link myriad systems across domains. HIT standards help this effort, but security policies to link the domains must also be addressed. However, we see this project's effort as another illustration of what can be achieved when a functional health information exchange has been established.

We believe this approach will bridge the last mile, at least until EMRs are in widespread use. Admittedly, the bridge is not ideal. Communication is asynchronous because a paper must be completed and faxed for each step in the communication. For use cases in which the data exchange must be faster, synchronous communication such as a real-time EHR connection, a web interface, or even a phone call will be necessary. In the future, the approach we have taken can be adapted to the delivery of electronic data directly to and from an EHR. In that case, the errors inherent in scanning paper, the additional manual effort to check the scans, and the need for fax machines will be eliminated. However, for a busy clinician without a linked EHR, logging into a web interface or making a phone call represents a diversion from the clinic workflow. Only a medium that fits into busy clinic workflow will be acceptable. This is why a tailored, faxable, machine interpretable paper interface (i.e., an ATAD) offers a viable option. 
The Last Mile: Using Fax Machines to Exchange Data between Clinicians and Public Health

\section{Acknowledgements}

This work was funded by grant number 1P01HK000077 from the Centers for Disease Control and Prevention.

\section{Corresponding Author}

Stephen M. Downs, MD, MS

460 West $10^{\text {th }}$ Street, HS1000

Indianapolis, IN 46202

Office: $317-278-0552$

Fax: 317-278-0456

E-mail: stmdowns@iupui.edu)

\section{References}

1. Thacker SB, Berkelman RL. 1988. Public Health Surveillance in the United States. Epidemiol Rev. 10, 164-90.

2. Elson RB, Connelly DP. 1995. Computerized Patient Records in Primary Care: Their Role in Mediating Guideline- Driven Physician Behavior Change. Arch Fam Med. 4, 698-705. http://dx.doi.org/10.1001/archfami.4.8.698

3. Yasnoff W, O’Carroll P, Koo D, Linkins R, Kilbourne E. 2000. Public Health Informatics: Improving and Transforming Public Health in the Information Age. J Public Health Manag Pract. 6(6), 67-75. http://dx.doi.org/10.1097/00124784-200006060-00010

4. Hinman A, Atkinson D, Diehn T, et al. Principles and Core Functions of Integrated Child Health Information Systems Journal of Public Health Management and Practice. 200410 S52S56.

5. Carroll AE, Downs SM. 2006. Comprehensive cost-utility analysis of newborn screening strategies. Pediatrics. 117(5 Pt 2), S287-95.

6. $\quad$ Kemper AR, Uren RL, Moseley KL, Clark SJ. 2006. Primary care physicians' attitudes regarding follow-up care for children with positive newborn screening results. Pediatrics. 118(5), 1836-41. http://dx.doi.org/10.1542/peds.2006-1639

7. U.S. Department of Health and Human Services. 2000. Office of Disease Prevention and Health Promotion--Healthy People 2010. Nasnewsletter. 15(3), 3.

8. Zimmerman R. AAFP, AAP and ACIP Release 1998 Recommended Childhood Immunization Schedule. 1998

9. Progress in immunization information systems --- United States, 2009. MMWR Morb Mortal Wkly Rep. Jan 14;60(1):10-12.

10. Leu MG, O'Connor K, Marshall R, Klein JD. Pediatricians' Use of Health Information Technology: A National Survey. Paper presented at: Pediatric Academic Societies Annual Meeting2010.

11. Kemper AR, Uren RL, Clark SJ. 2006. Adoption of electronic health records in primary care pediatric practices. Pediatrics. 118(1), e20-24. http://dx.doi.org/10.1542/peds.2005-3000 
12. Health information technology: initial set of standards, implementation specifications, and certification criteria for electronic health record technology. Final rule. Fed Regist. Jul 28;75(144):44589-44654.

13. Biondich PG, Grannis SJ. 2004. The Indiana network for patient care: an integrated clinical information system informed by over thirty years of experience. J Public Health Manag Pract. Suppl, S81-86. http://dx.doi.org/10.1097/00124784-200411001-00013 14.2010 Annual Report: Indiana Health Information Exchange;2011.

15. Barnes M. 2007. Lessons learned from the implementation of clinical messaging systems. AMIA Annu Symp Proc. $\bullet \bullet, 36-40$.

16. Anand V, Biondich P, Liu G, Rosenman M. SM D. Child Health Improvement through Computer Automation: The CHICA System. Paper presented at: MEDINFO 20042004 San Francisco.

17. Seebregts CJ, Mamlin BW, Biondich PG, et al. 2009. The OpenMRS Implementers Network. Int J Med Inform. 78(11), 711-20. http://dx.doi.org/10.1016/j.ijmedinf.2008.09.005

18. Wolfe BA, Mamlin BW, Biondich PG, et al. 2006. The OpenMRS system: collaborating toward an open source EMR for developing countries. AMIA Annu Symp Proc. •••, 1146.

19. Jenders RA, Hripcsak G, Sideli RV, et al. 1995. Medical decision support: experience with implementing the Arden Syntax at the Columbia-Presbyterian Medical Center. Proc Annu Symp Comput Appl Med Care. $\bullet \bullet, 169-73$.

20. Watson MS, Mann MY, Lloyd-Puryear MA, Rinaldo P, Howell RR. 2006. American College of Medical Genetics Newborn Screening Expert Group Newborn Screening: Toward a Uniform Screening Panel and System-Executive Summary. Pediatrics. 117(5), S296-307.

21. Zhu VJ, Overhage MJ, Egg J, Downs SM, Grannis SJ. 2009. An empiric modification to the probabilistic record linkage algorithm using frequency-based weight scaling. J Am Med Inform Assoc. 16(5), 738-45. http://dx.doi.org/10.1197/jamia.M3186

22. Davidson K. Adapting Vendor Clinical Systems for Real-Time Registry Participation Using HL7. National Immunization Registry Conference. Vol Atlanta, GA2003.

23.Downs SM, van Dyck PC, Rinaldo P, et al. Improving newborn screening laboratory test ordering and result reporting using health information exchange. J Am Med Inform Assoc. JanFeb;17(1):13-18.

24. Newborn Screening AHIC Detailed Use CaseWashington. DC: U.S. Department of Health and Human Services Office of the National Coordinator for Health Information Technology; 2008

25. Spivak L, Dalzell L, Berg A, et al. 2000. New York State universal newborn hearing screening demonstration project: inpatient outcome measures. Ear Hear. 21(2), 92-103. http:// dx.doi.org/10.1097/00003446-200004000-00004

26. Kim S, Lloyd-Puryear MA, Tonniges TF. 2003. Examination of the communication practices between state newborn screening programs and the medical home. Pediatrics. 111(2), E120-26. http://dx.doi.org/10.1542/peds.111.2.e120

27. Centers for Disease Control and Prevention, National Immunization Survey. 2011; http:// www.cdc.gov/nchs/nis/data files.htm. Accessed August 14, 2011. 